\title{
Next Generation Genetic Algorithm for Maximum Clique Problem
}

\author{
Savita, Sudha \\ ${ }^{1}$ Computer Science, Aitm/Mdu, India) \\ ${ }_{2}^{2}$ (Assistant Professor Cse Dept. Aitm/Mdu,India)
}

\begin{abstract}
Given a graph, in the maximum clique problem, one desires to find the largest number of vertices, any two of which are adjacent. A branch-and-bound algorithm for the maximum clique problem-which is computationally equivalent to the maximum independent (stable) set problem-is presented with the vertex order taken from a coloring of the vertices and with a new pruning strategy. The algorithm performs successfully for many instances when applied to random graphs and DIMACS benchmark graphs.
\end{abstract}

Keywords: Maximum clique problem,planner graphs,Heuristics, Genetic Algorithm,NP hard.

\section{Introduction}

The introduction of the paper should explain the nature of the problem, previous work, purpose, and the contribution of the paper. The contents of each section may be provided to understand easily about the paper. The clique problem refers to any of the problems related to finding particular complete sub graphs ("cliques") in a graph, i.e., sets of elements where each pair of elements is connected.

For example, the maximum clique problem arises in the following real-world setting. Consider a social network, where the graph's vertices represent people, and the graph's edges represent mutual acquaintance. To find a largest subset of people who all know each other, one can systematically inspect all subsets, a process that is too time-consuming to be practical for social networks comprising more than a few dozen people. Although this brute-force search can be improved by more efficient algorithms, all of these algorithms take exponential time to solve the problem. Therefore, much of the theory about the clique problem is devoted to identifying special types of graph that admit more efficient algorithms, or to establishing the computational difficulty of the general problem in various models of computation. ${ }^{[1]}$ Along with its applications in social networks, the clique problem also has many applications in bioinformatics and computational chemistry.

Clique problems include:

- $\quad$ finding the maximum clique (a clique with the largest number of vertices),

- finding a maximum weight clique in a weighted graph,

- $\quad$ listing all maximal cliques (cliques that cannot be enlarged)

- $\quad$ solving the decision problem of testing whether a graph contains a clique larger than a given size.

These problems are all hard: the clique decision problem is NP-complete (one of Karp's 21 NPcomplete problems), the problem of finding the maximum clique is both fixed-parameter intractable and hard to approximate, and listing all maximal cliques may require exponential time as there exist graphs with exponentially many maximal cliques. Nevertheless, there are algorithms for these problems that run in exponential time or that handle certain more specialized input graphs in polynomial time.

Although complete sub graphs have been studied for longer in mathematics, ${ }^{[3]}$ the term "clique" and the problem of algorithmically listing cliques both come from the social sciences, where complete sub graphs are used to model social cliques, groups of people who all know each other. The "clique" terminology comes from Luce \& Perry (1949), and the first algorithm for solving the clique problem is that of Harary \& Ross (1957), ${ }^{[1]}$ who were motivated by the sociological application.

\section{A Next Generation Genetic Algorithm (Ngga) For Maximum Clique Problem}

In this section, a next generation genetic algorithm for solving the maximum clique problem (NGGAMC) is presented. The NGGAMC basically follows the steps of a steady-state genetic algorithm, which means only a portion of the population is replaced at each generation. If the whole population is replaced at each generation, it is called a generational GA. the main features of the NGGAMC are a strong local optimizer to accelerate the convergence and the adaptive genetic operators to fine tune the algorithm. Also, graph preprocessing is used before creating the initial population to help keep potentially good partial solutions from being disrupted by crossover. This algorithm is a modified genetic algorithm for solving the maximum clique 
problem .In this work preserved the good features of their algorithm such as preprocessing the input graph and using local optimization, but modified the local optimization algorithm, the genetic operators such as crossover and mutation to make them adaptive. Also, the methods of extracting a clique is straight forward with more efficiency and enlarging a clique by randomizing the process of deleting or adding vertices.

\subsection{Proposed Algorithm}

Input: $\mathrm{G}=(\mathrm{V}, \mathrm{E})$

Output: a maximal clique in $\mathrm{G}$

Preprocess the input graph

Create an initial population

Apply the local optimization to each chromosome

While (stopping condition is not met) do

Select two parents, p1 and $\mathrm{p} 2$, from the population generate two offspring by crossing over $\mathrm{p} 1$ and $\mathrm{p} 2$

Mutate the two offspring Local optimize the two offspring

Replace a population member with a better offspring update stopping condition End while

Return the best member of the population

A next Generation genetic algorithm (NGGA) requires the genetic representation of the solution domain and the fitness function to evaluate the resolution domain. Once the genetic representation and the fitness function are defined, a NGGA proceeds to initialize a population of solution $\&$ then to improve it.

\subsection{Maximum Clique Problem}

\section{Figures and Tables}

Figure 1.1 FIGURE 1.1 The graph shown has one maximum clique, the triangle $\{1,2,5\}$, and four more maximal cliques, the pairs $\{2,3\},\{3,4\},\{4,5\}$, and $\{4,6\}$.

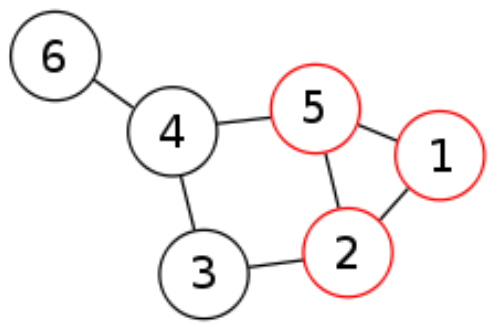

3.2 Special Classes Of Graphs

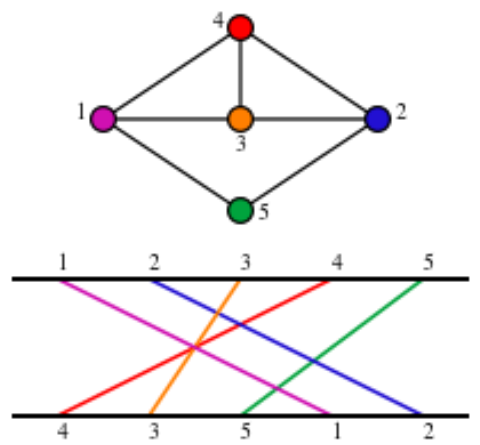

Figure 1.2 In this, the maximum cliques correspond to the longest decreasing subsequences $(4,3,1)$ and $(4,3,2)$ of the defining graph.

\subsection{Applications Of Genetic Algorithm}

GA have been used in a large number of scientific and engineering problems and models. Some examples are briefly introduced below.

- Optimization: gas have been used in a variety of optimization tasks, including numerical optimization and combinatorial optimization problems such as the traveling salesman problem and the graph partitioning Problem.

- $\quad$ Automatic programming: gas have been used to evolve computer programming for specific tasks, and to design other computational structures such as cellular automata and sorting networks. This technique becomes a branch of evolutionary computation area, called genetic algorithm. 


\section{Implementation}

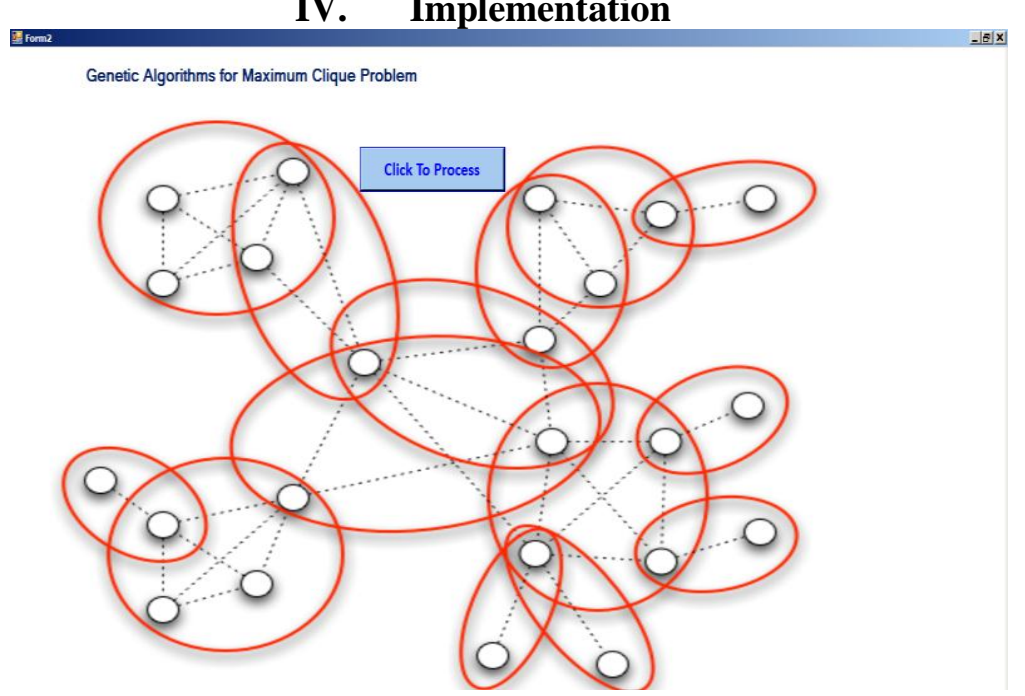

Figure 4.1 : Staring Interface to get Maximum Clique
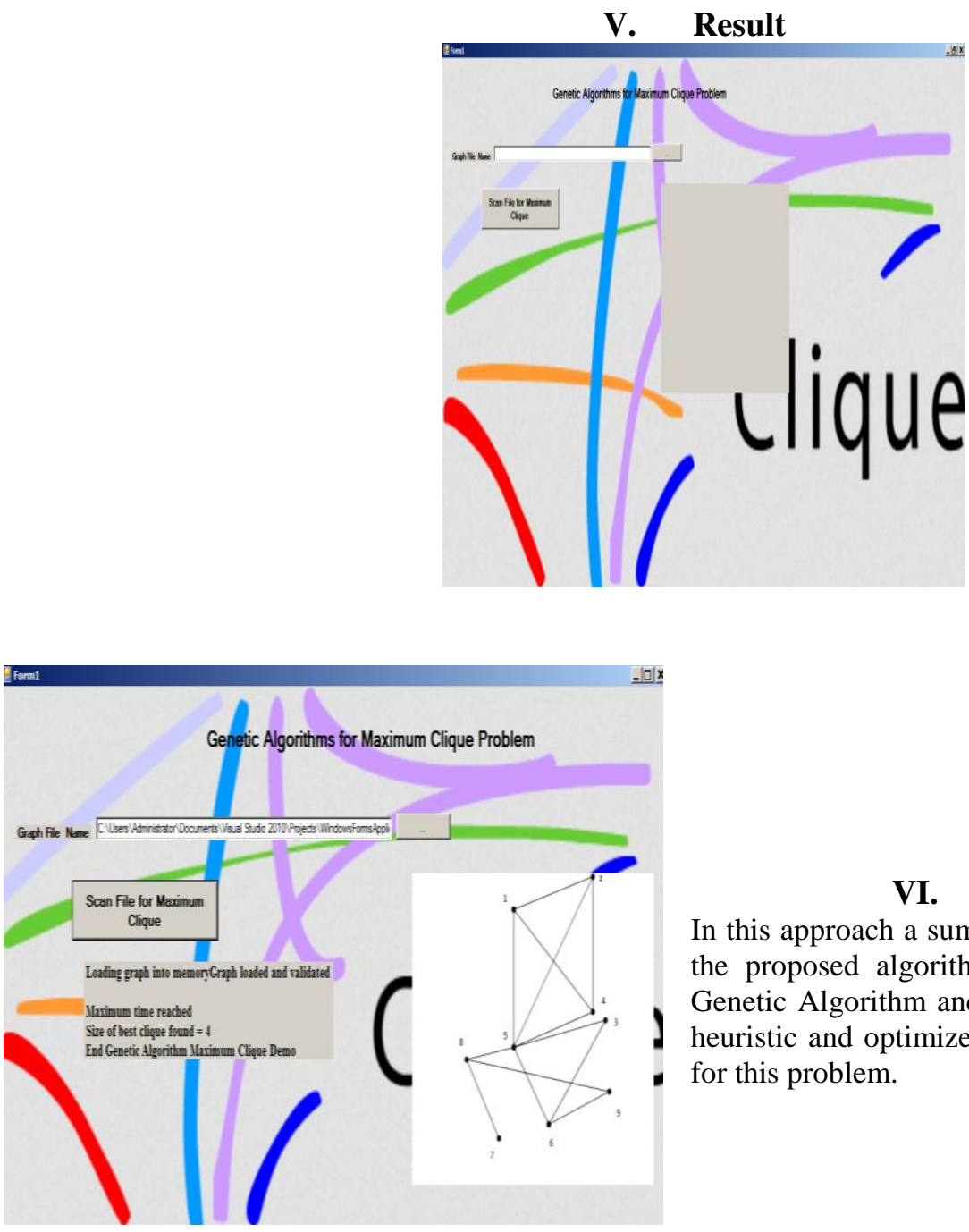

- $] \underline{x}$

Figure 4.1 : Maximum Clique For the first Clique File V.2 


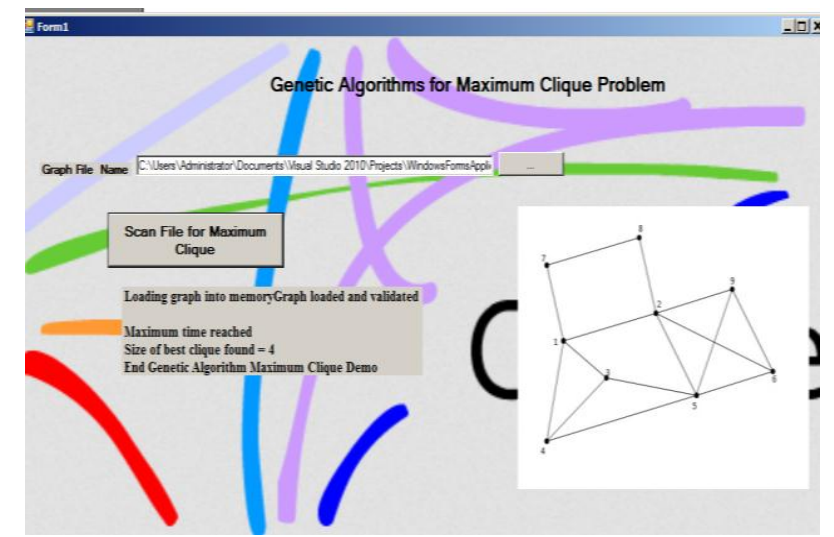

Figure 4.3: Maximum Clique For the second Clique File

\section{Acknowledgements}

I hereby declare that this submission is my own work and that, to the best of my knowledge and belief, it contains no material previously published or written by another person nor material which has been accepted for the award of any other degree or diploma of the university or other institute of higher learning, except where due acknowledgment has been made in the text.

[1]. Cazals \& karande(2008);Eppstein \& Strash(2011).

\section{References}

[2]. Eppstein;Loffler \& Strash (2010).

[3]. Regin (2003)

[4]. Tomita \& Kameda (2007);Konc \& Janezic (2007)

\section{Proceeding Papers:}

Paper: Finding Maximum Clique With A Genetic Algorithm

Author: BO HUANG

Date of Conference: April, 2002 